\title{
ELECTROPHYSIOLOGICAL CORRELATES OF SUICIDALITY
}

\author{
Tomáš Rakús ${ }^{1,2}$, Katarína Hubčíková ${ }^{1,2}$, Lucia Bruncvik ${ }^{2,3}$, \\ Zuzana Pechanová ${ }^{1,2}$ \& Martin Brunovský ${ }^{2,4}$ \\ ${ }^{I}$ Department of Neuropsychiatry of Slovac Medical University in Bratislava and Psychiatric Hospital \\ of Philippe Pinel in Pezinok, Pezinok, Slovakia \\ ${ }^{2}$ Third faculty of medicine Charles University in Prague, Czech republic \\ ${ }^{3}$ Landesklinikum Hainburg, Austria \\ ${ }^{4}$ National Institute of mental health Czech republic, Klecany, Czech republic
}

received: 10.2.2021;

revised: 9.4.2021;

accepted: 23.5 .2021

\section{SUMMARY}

Suicidal risk assessment is still a major challenge not only in psychiatric practice. Clinical investigation of suicidality can be significantly improved by using standardized scales for assessing suicide risk. The choice of a method for assessing suicidality also has significant implications for the search of valid available biomarker of suicidal behavior, where a less complex suicidality assessment procedure yields inaccurate results. This article offers an overview and analyzes in detail clinical studies of suicidality by electrophysiological methods since 2005 to 5/2020, especially in connection with presumed pathophysiological mechanism of the „suicidal brain" and the chosen method of sucidality assessment. Electrophysiological methods such as quantitative electroencephalography indicators, event-related potential, loudness dependence of the auditory evoked potential, polysomnography and heart rate variability offer a robust battery of easily available methods for assessing impaired emotional regulation. Nowadays it is unfortunately very difficult to point out the optimal electrophysiological examination of suicidal behaviour because of conflicting conclusion of presented studies which have been probably caused by various suicidal risk assessments, not always available data on affecting medication prior to testing and small samples of suicidal participants among studies. The most consistent and hopeful results are presented by evaluation of theta power by quantitative electroencephalography, although there are also few conflicting conclusions. The authors of this paper believe that this article could be good starting point for further research of electrophysiological methods in the field of suicidality.

Key words: suicidality - quantitative electroencephalography - loudness dependence of the auditory evoked potential - polysomnography - heart rate variability

\section{INTRODUCTION}

Suicide is the fourteenth most common cause of death globally, but it is one of the most common causes of death in young people aged 15-44, with approximately 20 suicide attempts per suicide commited (WHO 2007). Evaluation of suicidal symptomatology, whether in terms of assessing the relevance of the risk of escalation to severe suicidal actions, or in terms of optimal therapeutic management (most often, but not only) in chronically suicidal patients, is still a major challenge in psychiatric practice.

Despite the various available assistive methods, a significant number of psychiatrists (53\% of workplaces in the Czech Republic and Slovakia (Rakús \& Pechanová 2018)) consider a long-term comprehensive examination by an experienced doctor without the use of any other tests to be the best diagnostic tool. On the other hand, the superiority of the clinical opinion of experienced psychiatrists over a standard psychometric test evaluating suicide - specifically The Columbia Suicide Severity Rating Scale (C-SSRS) (Posner et al. 2011) - in predicting suicidal attempts or completed suicides was not demonstrated in a prospective study, both methods were equivalent in suicidal attempts prediction, the Colombian scale yielded better results at commited suicides predicition (Randal et al. 2018).

However, the possibilities of assessing their predictive ability are somewhat limited by the fact that a commited suicide is, fortunately, a relatively rare phenomenon in most healthcare facilities (Perlman et al. 2011). Nevertheless, they provide useful information on the severity and complexity of the level of suicidal risk, although it needs to be noted, that sometimes the individual items assessed (e.g. a developed suicidal plan) are more important than the overall score (Perlman et al. 2011). In particular, the authors of this paper would like to emphasize the detailed investigation of suicidal behavior as offered by the The Columbia Suicide Severity Rating Scale (Posner et al. 2011), which, in addition to the traditional classification of suicidal behavior, stratifies suicidal behavior into more detailed categories. These are: self-harm behavior without a suicidal intention, aborted attempt (the person is disturbed by an external circumstance when a potential selfharm is initiated), prematurely terminated attempt (the person stops during a suicide attempt) and preparatory actions or behavior (actions leading to suicidal attemps, that go beyond mere thoughts or preparing for death by suicide, such as writing a farewell letter). 
The choice of a method for assessing suicidality also has significant implications for examining suicidality in an effort to find a valid biomarker of suicidal behavior when less complex scales (e.g. without a clearly defined suicidal attempt) yield inaccurate and limited results (Park 2014). According to a recent review article (BaniFatemi et al. 2018), functional neuroimaging (positron emission tomography, functional magnetic resonance) and structural neuroimaging methods (computed tomography, magnetic resonance imaging, diffuse tensor imaging) point to dysfunction of fronto-temporal networks with volume reduction of grey and white matter in the prefrontal cortex, anterior cingulate, and upper anterior temporal gyrus of a suicidal brain across psychiatric diagnoses (major depression disorder, bipolar disorders, psychotic disorders, borderline personality disorder). These findings correlate with the presumption of executive dysfunction with a direct impact on emotional regulation in individuals with suicidal behavior, where regulatory emotional strategies are disrupted and maladaptive procedures with reduced ability to cope with emotional stress are used instead (Jollant et al. 2011). However, the introduction of these methods for the detection of potential suicidal patients into a routine clinical practice is problematic, not only in view of their economic costs.

On the other hand, more advanced electrophysiological methods - such as quantitative electroencephalography (QEEG), with the application of a modern mathematical analysis with current computer processing capabilities, are able to approach (functional) neuroimaging methods by a relative good spatial resolution and with excellent time resolution (on a millisecond scale), and with minimal economic costs they are easily replicable and remove the need to administer a radioactive substance or generate a strong magnetic field (Hughes \& Johne 1999). Frontal QEEG changes in suicidality studies (Kopeček et al. 2007, Iosifescu et al. 2008, Hunter et al. 2010, Lee et al. 2017, Park et al. 2019) - especially changes in theta activity $(4-8 \mathrm{~Hz})$, which is closely related to anterior cingulate activity (Schiller 2019) appear to be a promising correlate of suicidal symptoms, either by assessing absolute/relative power, (a)symmetry (the ratio of power in each band between symmetrical pairs of left and right hemisphere electrodes (Hughes \& Johne 1999)), coherence (degree of synchronization between two channels), cordance (allows to assess the integrity of afferent inputs in one brain area (Leuchter et al. 1994)) or using LORETA (Low Resolution Brain Electromagnetic Tomography). However, it should be noted, that non-invasive quantitative electroencephalography determines the distribution of actual neuronal electrical activity of the brain in space, which is limited to the area of cortical gray matter and hippocampi, as subcortical structures such as basal ganglia and brainstem nuclei act as closed dipoles, whose electrical potentials do not reach the surface of the head and therefore cannot be sensed by surface electrodes, resp. the signals are limited (Attal et al. 2013).
Another pathophysiological mechanism of suicidal behavior is central serotonergic dysfunction, which has been demonstrated in postmortem studies of commited suicides (Mann et al. 2000, Pandey et al. 2002), genotyping research of the serotonin transporter gene (Lee et al. 2015) or PET/SPECT visualization 5-HT receptors or serotonin transporter binding potential (Paterson et al. 2013). Central serotonergic activity can also be examined non-invasively by Loudness Dependence of Auditory Evoked Potentials (LDAEP), which reflect low levels of serotonin in the CNS by a strong dependence of the amplitude of the evoked potential on loudness and vice versa (Hegerl \& Juckel 1993).

Prefrontal ventromedial cortex with overnight beta activity is also involved in the process of sleep regulation based on PET-FDG studies of dyssomnia in depressed patients (Nofzinger 2004). Intermediate fastfrequency EEG activity (alpha and beta) during sleep appears to be an indicator of excessive central nervous system irritability and may correlate with the severity of depressive disorders (Cheng 2015). Studies of polysomnographic examinations or quantitative sleep EEGs point to differences in sleep architecture and dream activity of depressed patients at suicidal risk compared to depressed patients without suicidal risk (Agargun et al. 2003, Ballard et al. 2016, Bernert et al. 2017, Dolsen et al. 2017, Boafo et al. 2019).

The presumed problematic emotional regulation, that may increase the risk of suicidal behavior (Rajappa et al. 2012) can be influenced and investigated by cognitive reassessment, i.e. a cognitive strategy of re-framing emotional stimuli to change the intensity, length or polarity of emotion (Gross et al. 2003). Deviations in the selective distraction from other stimuli to suicide-specific information and vice versa can be interpreted as a cognitive vulnerability to suicide (Wenzel et al. 2008). The importance of problematic distraction from suicidally relevant stimuli can be examined, resp. it is investigated using cognitive evoked potentials (Kudinova et al. 2016, Baik et al 2018).

Impaired flexibility of emotional regulation can also be examined using heart rate variability and respiratory sinus arrhythmia - by measuring cardiac performance, which is also controlled by the corticolimbic system (Myers 2016). Studies of high-frequency heart rate variability (Adolph et al. 2018, Wilson et al. 2016) and respiratory sinus arrhythmia (Tsypes et al. 2015, Yang et al. 2019) indicate a reduced capacity to regulate the emotional response to stress in patients with suicidal symptoms.

The aim of this paper is to provide summary of electrophysiological methods as a potential biomarkers of suicidal risk.

An overview of the presented electrophysiological methods in the context of pathophysiological mechanisms of the suicidal brain is illustrated in Figure 1. 

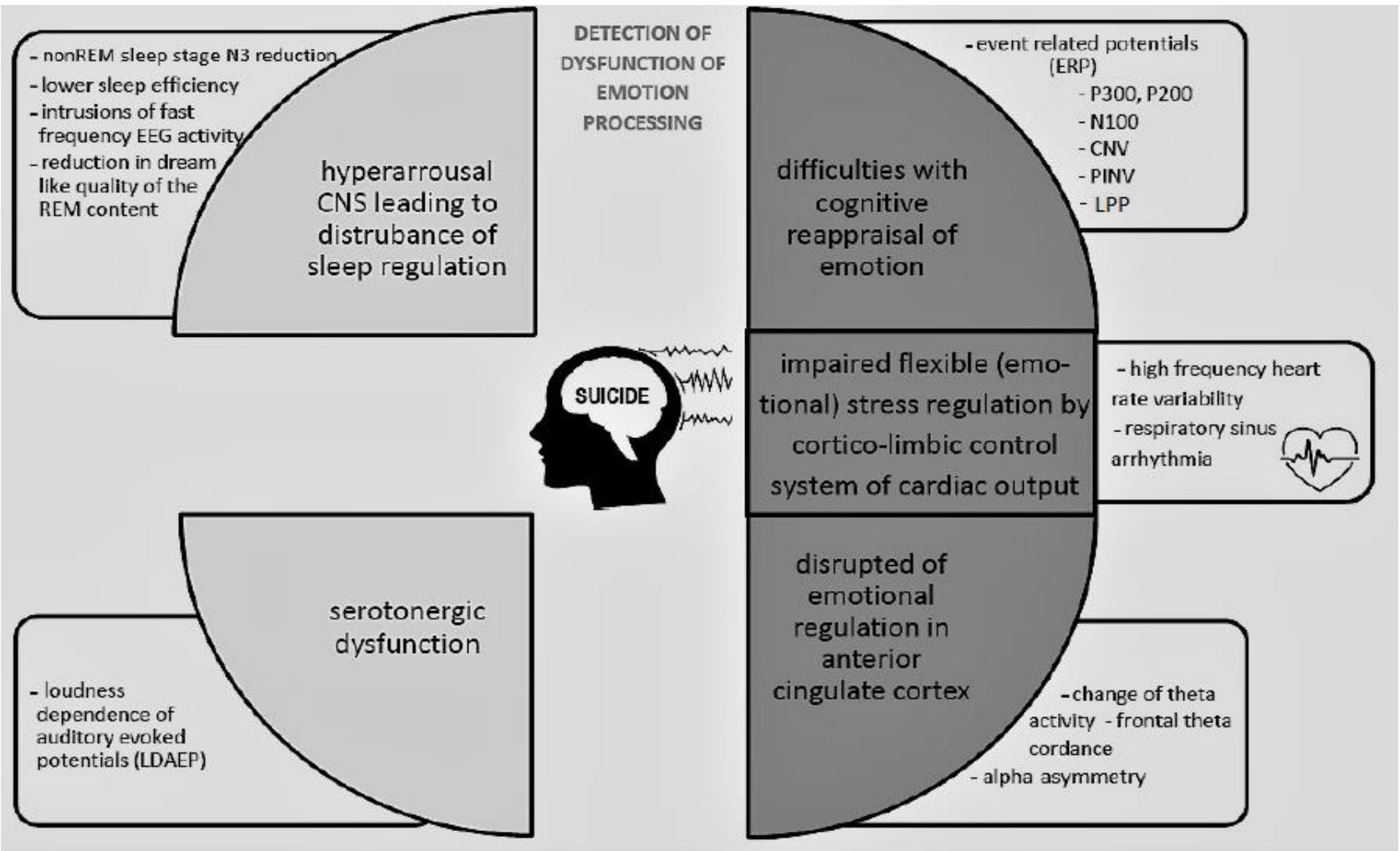

EEG - electroencephalography, REM - rapid eye movement, CNS - central nervous system, CNV - contingent negative variation, PINV postimperative negative variation, LPP - late positive potential

Figure 1. Overview of electrophysiological methods in context of pathophysiological mechanisms of suicidal brain

\section{METHODS}

The following key words were used in pubmed: „quantitative electroencephalography“, „polysomnography“, „event related potentials“, „LDAEP“, „, heart rate variability“ and ,suicid*“. Articles abstracts were all manually inspected as an additional search to see if they were within the scope of the review, next references lists of these chosen studies were scanned and relevant studies were added manually. We included only articles in which the association between suicidality and electrophysiological measurement was directly tested. Articles not written in english were excluded. There were 130 articles identified in initial search, using the parameters specified in the method we were able to identify 33 articles that met the predetermined inclusion criteria. Specifically our review classifies the studies considering the following levels: amount of probands, medication before electrophysiological assessment, assessment of suicidality.

\section{RESULTS}

This paper offers an overview of clinical studies of suicidality by electrophysiological methods, namely quantitative electroencephalography, loudness dependence of auditory evoked potentials, event-related potentials, polysomnography/quantitative sleep EEG and heart rate variability, published since 2005 to the 5/2020. The findings are summarised in Tables 1 to 3 .

\section{Quantitative electroencephalography}

The principle of quantitative electroencephalography lies in precise and objective quantification of electrophysiological brain activity by computerized analytic and statistical techniques. Obviuosly the technique include visual, semi-automatic or automatic selection of several artifact-free epochs of multichannel EEG (usually 1-2 minutes in length in total), which are then analyzed using usually a fast Fourier transform (a mathematical basis for analyzing performance spectra), resulting in an estimate of different frequencies (Hughes \& Johne 1999). The results from each electrode can then be further processed by various QEEG tools:

- absolute/relative (percentage of total performance in each channel) performance in each band;

- coherence - the degree of synchronization of 2 signals sensed from different places of the scalp; can be interpreted as functional connectivity of different areas of the brain (Thatcher et al. 1986);

- (a)symmetry - the ratio of performance in each band between symmetrical pairs of electrodes from the left and right hemispheres (Hughes \& John 1999);

- cordance - a complex measurement of the performance spectra of each of the surface electrodes, which puts both of them into a mutual relationship, i.e. absolute and relative performance spectra (Leuchter et al. 1994); has a strong correlation with brain perfusion measured by PET (Leuchter et al. 1999) and SPECT (Cook et al. 1998); 


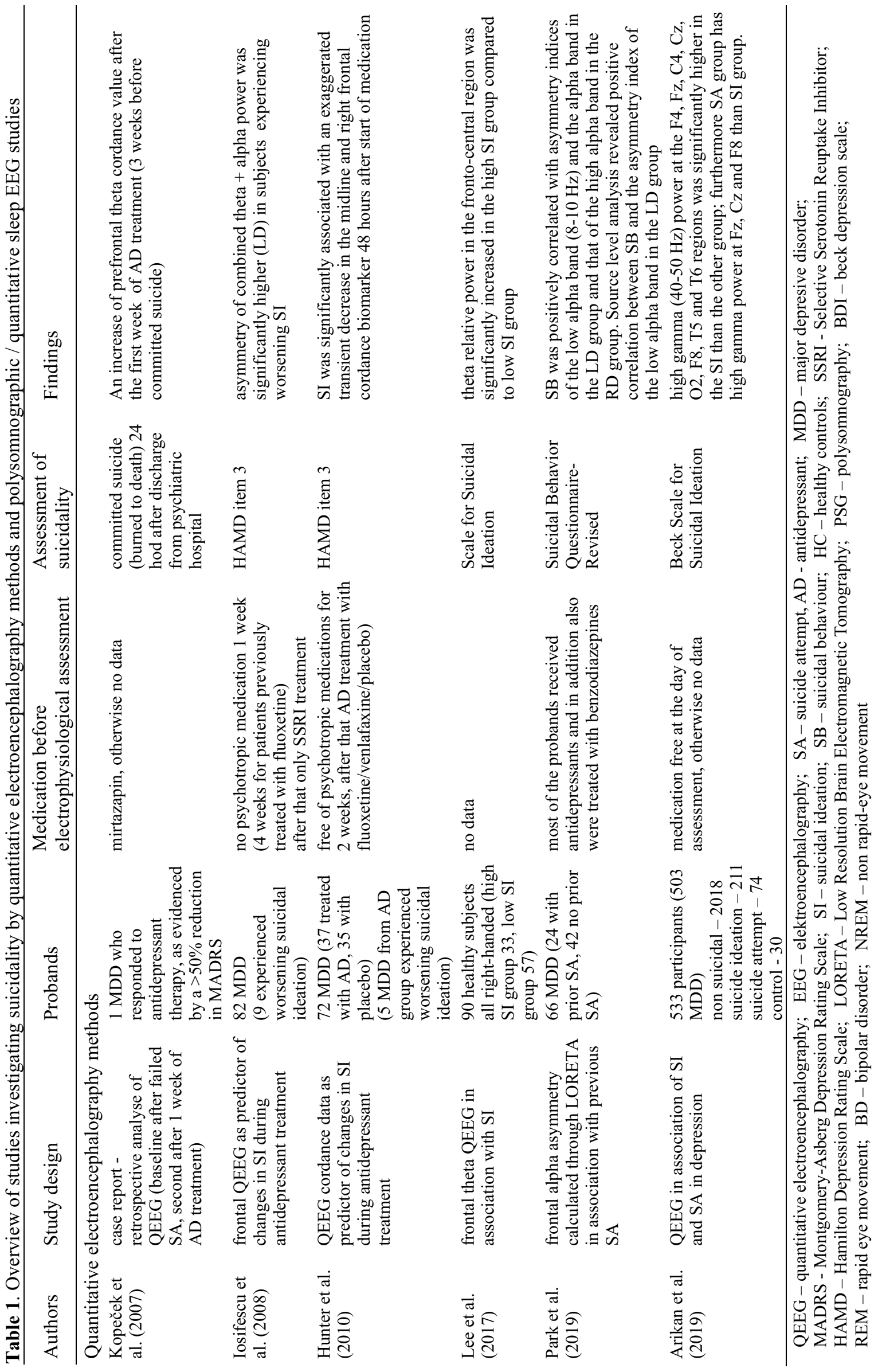




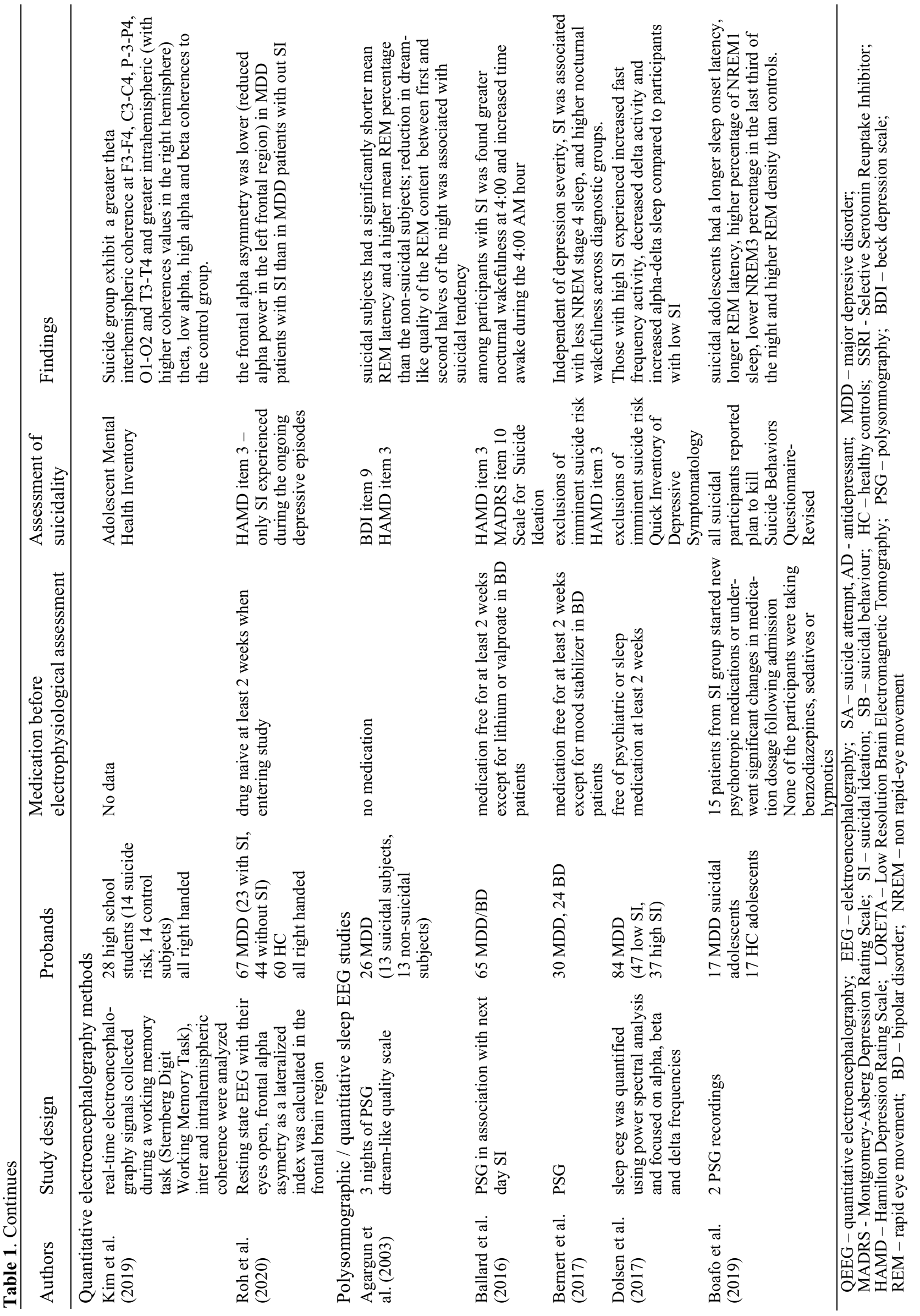




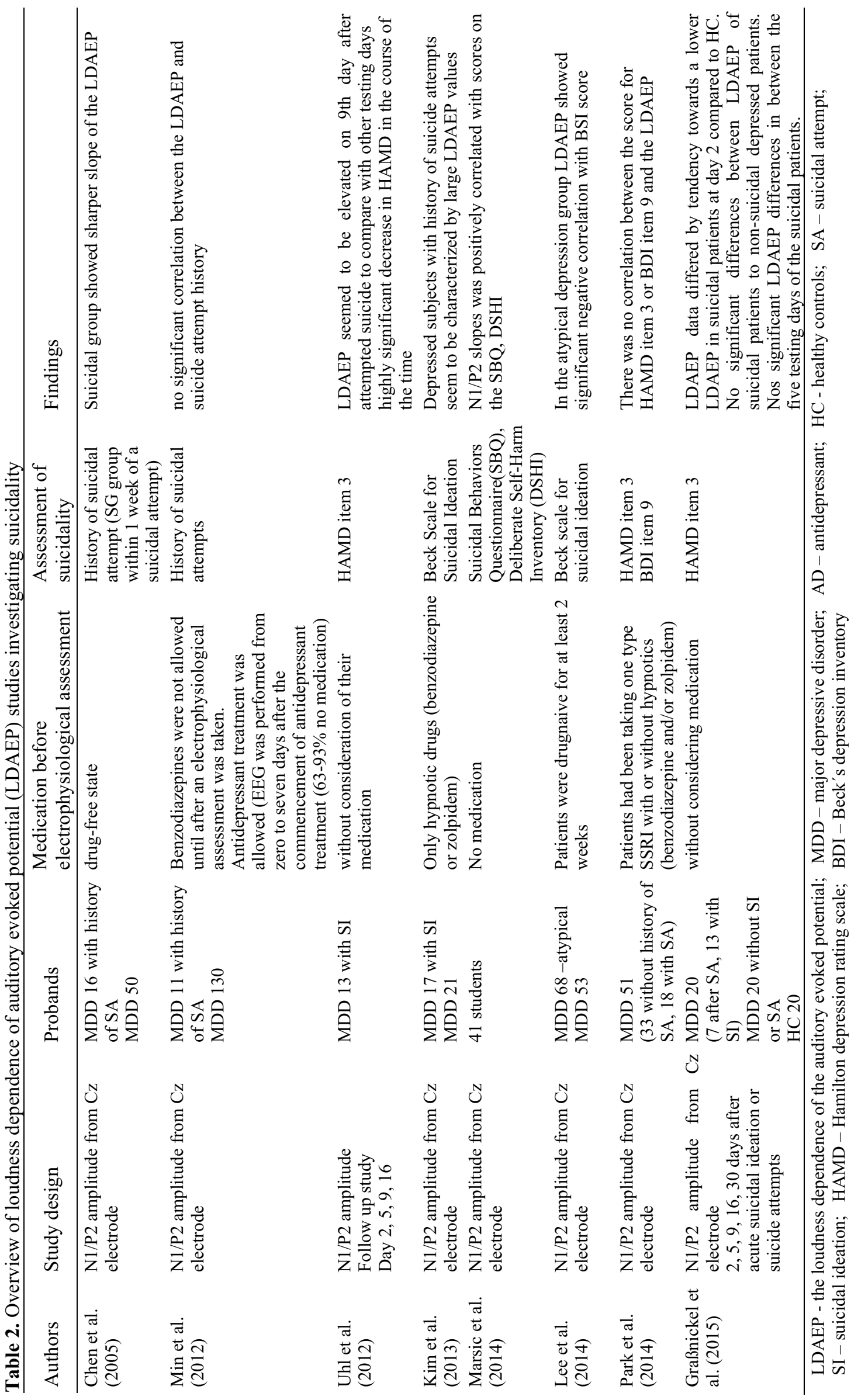




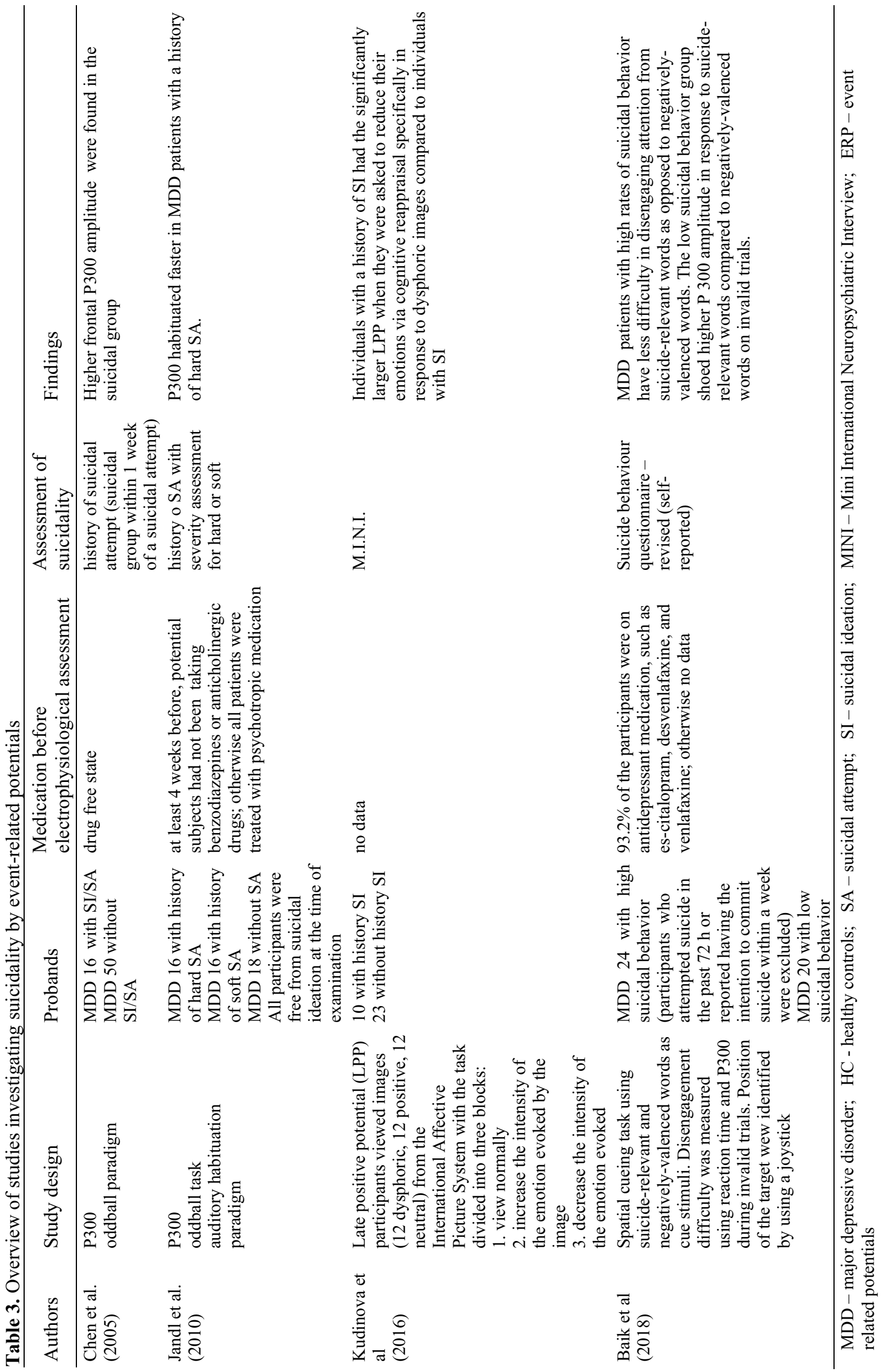




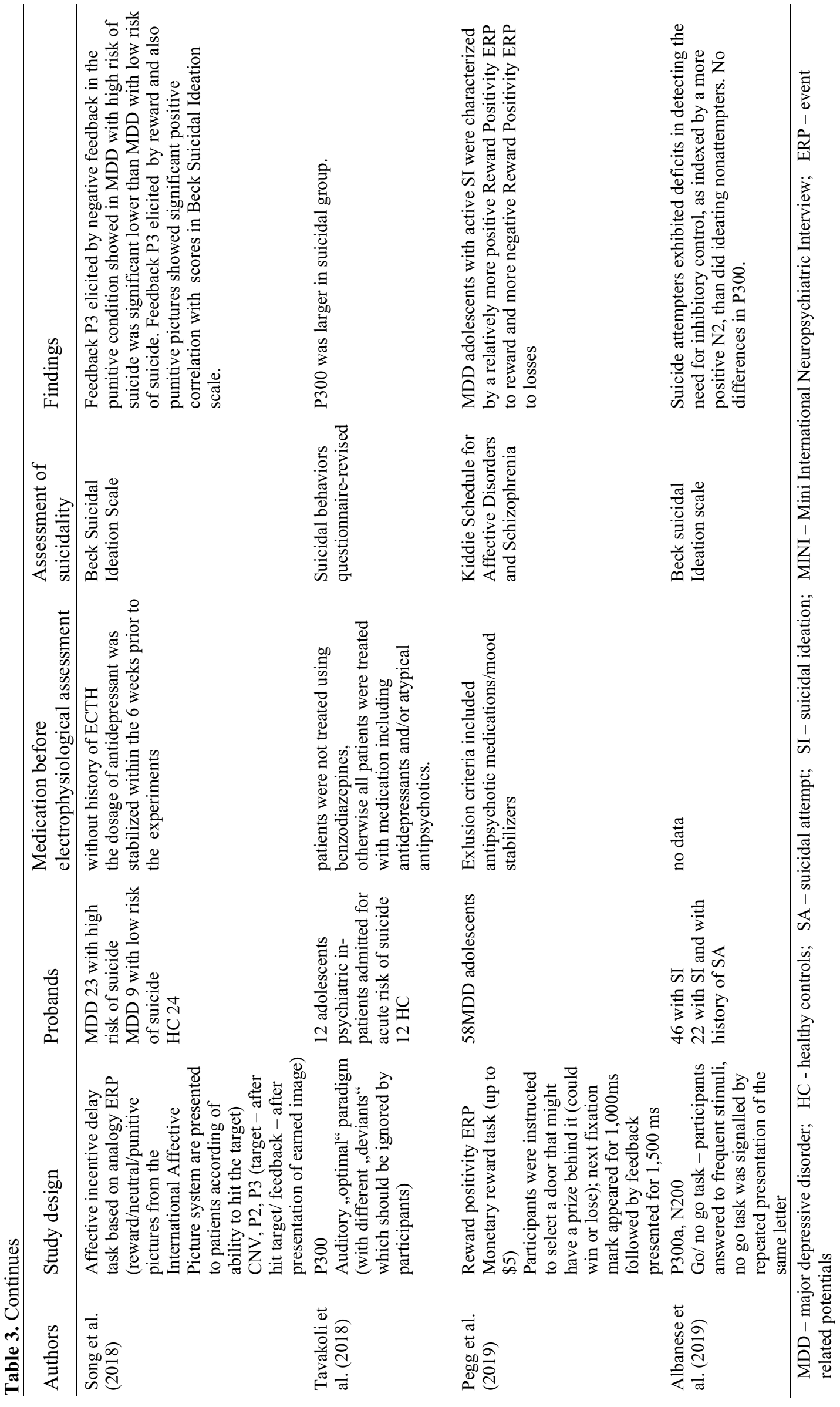


- LORETA (Low Resolution Brain Electromagnetic Tomography) - a functional neuroimaging method that is based on electrophysiological and neuroanatomical assumptions and limitations of noninvasive localization of neuronal generators responsible for the emergence of EEG phenomena (Pasqual Marqui 2002a,b, 2011).

Frontal changes in QEEG in the study of suicidality, especially an increase in theta activity $(4-8 \mathrm{~Hz})$, which is closely associated with anterior cingulate activity (Schiller 2019), was confirmed by several studies higher theta cordance associated with suicidal ideas (Lee et al. 2017), higher theta cordance detected by retrospective analysis after complete suicide with dissimulated clinical signs (even using psychometric scales!) 24 hours before burning themselves (Kopeček et al. 2008), higher theta interhemispheric coherence not only frontally in the suicidal group (Kim et al. 2019). In contrast, the decrease in theta cordance in association with suicidal symptoms is confirmed by Hunter et al. (2010). The asymmetry of the performances of the individual bands was also investigated, while the asymmetry of the combined theta and alpha power was in the study of Ioniflescu et al. (2008) higher with worsening of suicidal symptoms, in contrast Roh et al. (2020) describe lower frontal alpha asymmetry associated with suicidal symptoms.

\section{Polysomnographic examination and quantitative sleep EEG}

Intrusions of fast-frequency activity (alpha and beta) during sleep appears to be an indicator of excessive central nervous system excitability and may correlate with the severity of depressive disorders (Cheng 2015). Studies of polysomnographic examinations or quantitative sleep EEGs point to differences in the sleep architecture and dream activity of depressed patients at suicidal risk and depressed patients without suicidal risk (Agargun et al. 2003, Ballard et al. 2016, Bernert et al. 2017, Boafo et al. 2019). Suicidal individuals have a significantly shorter REM latency, a higher proportion of REM sleep (Agargun et al. 2003), less NREM 3-4 stages (Boafo et al. 2019, Bernert et al. 2017), a reduction in dream REM quality activity (Agargun et al. 2003) and more frequent nocturnal awakenings (Ballard et al. 2016). Quantitative evaluation of sleep EEG showed increased fast frequency activity, decreased delta activity, and increased alpha - delta sleep compared to patients with less pronounced suicidal ideas (Dolsen et al. 2017).

\section{The loudness dependence of the auditory evoked potential - LDAEP}

Central serotonergic activity has been shown to be associated with suicidal symptoms (Paterson et al. 2013, Lee et al. 2015) and can be investigated non-invasively by the loudness dependence of the auditory evoked potential (LDAEP) with a strong LDAEP thought to reflect a weak serotonergic activity and vice versa (Hegerl \& Juckel 1993). The neurobiological model of the loudness dependence of the auditory evoked potential (LDAEP) in the primary auditory cortex is shown in Figure 2 (adapted from Juckel 2005). The primary auditory cortex is innervated predominantly by serotonergic (5-HT) neurons located in the nucleus raphe dorsalis of the reticular brainstem formation. Serotonergic activity from raphe dorsalis modulates pyramidal cells in the primary auditory cortex via 5$\mathrm{HT}_{1 \mathrm{~A}}$ (inhibitory) and 5- $\mathrm{HT}_{2}$ (excitatory) presynaptic receptors and indirectly via GABAergic interneurons. This serotonin modulating effect affects the reactivity of pyramidal cells during acoustic signal processing and at the same time acts on excitatory and inhibitory postsynaptic potentials (EPSP/IPSP) (Juckel 2005).

LDAEP is calculated as the ratio of the amplitudes of the evoked N1/P2 components in response to different intensities of sound stimuli (Hegerl et al. 2001). The formation of the N1/P2 complex and its distribution on the scalp is explained by two dipoles of each hemisphere - a tangentially oriented dipole reflects the activity of the primary auditory cortex, the radial dipole reflects the activity of the secondary cortex (Hegerl et al. 1993). However, as the primary auditory cortex shows greater 5-HT innervation (Juckel et al. 2003), some authors consider it crucial to evaluate LDAEP only from the primary cortex area to avoid overlapping signals from other areas and distorting evoked potential (Braverman et al. 2016). Therefore, a dipole source analysis (DSA) method is recommended that decomposes the surface sensed activity into said dipoles (Hegerl et al. 1993) or the use of voxel current density in 3D imaging using low resolution brain electromagnetic tomography (LORETA) (Pascal-Marqui 1994, Bravermanová et al. 2016). Most often, however, the authors derive LDAEP from only one electrode, most often from the $\mathrm{Cz}$ electrode (Henschl et al. 2008).

Studies indicate lower serotonergic activity (i.e. higher LDAEP) in depressed patients with suicidal symptoms than in depressed patients without suicidal risk (Chen et al. 2005, Kim et al. 2013, Marsic et al. 2014). On the contrary, this result is refuted by Graßnickeel et al. (2015), where higher serotonergic activity was observed in suicidal patients. Another approach is presented by Uhl et al. (2012), which monitors the dynamics of LDAEP over time with the highest LDAEP on day 9 after a suicidal attempt. Min et al. (2012) and Park et al. (2014) found no corelation between LDAEP and suicidality.

\section{Event-related potentials}

Event-related potentials (ERP) make it possible to investigate the problematic distraction from suicidally relevant stimuli (Kudinova et al. 2016, Baik et al. 2018). This presumed problematic emotional regulation, which may increase the risk of suicidal behavior (Rajappa et al. 2012), is influenced and investigated by cognitive 
reassessment, a cognitive strategy of framing emotional stimuli that changes the intensity, length or polarity of emotions (Gross et al. 2003). Deviations in the selective distraction from other stimuli to suicide-specific information and vice versa can be interpreted as a cognitive vulnerability to suicide (Wenzel et al. 2008).

The most explored ERP is the P300 wave, which is a potential complex of positive and negative waves with a dominant positivity of latency of about $300 \mathrm{~ms}$ from the moment of stimulation; it is often referred to as the neurophysiological correlate of the completion of the process of correct detection of a known stimulus and is independent of the modality of the stimulus used (Cigánek 1991). The most common paradigm in ERP stidies include "oddball", when two types of tones are detected. First one is common, so-called frequent tone and the second tone is rare, so-called target. The task of the examined person is to recognize target stimuli and count their number mentally (Cigánek 1991). Another paradigm is the "go-no go" paradigm, where the examined person performs an agreed action after detecting the target stimulus, e.g. press the button. In ERP suicidality studies, the P300 wave is most often used, which in suicidal subjects has a higher amplitude in the oddball paradigm compared to non-suicidal subjects (Chen et al. 2005, Tavakoli et al. 2018), or during a task with suicidally relevant words (Baik et al. 2018). In contrast, Albanese et al. (2019) in the go-no go paradigm found no difference in P300, but a more positive N2 amplitude of the suicidal group compared to the non-suicidal one. The lower feedback of P300 induced by punitive images in participants at high risk of suicide was pointed out by the study of Song et al. (2018). Among other ERPs, late positive potential (LPP) was also examined, which was significantly higher in participants with a history of suicidal ideation during the task of reducing negative emotion in response to dysphoric images compared to the non-suicidal group (Kudinova et al. 2016).

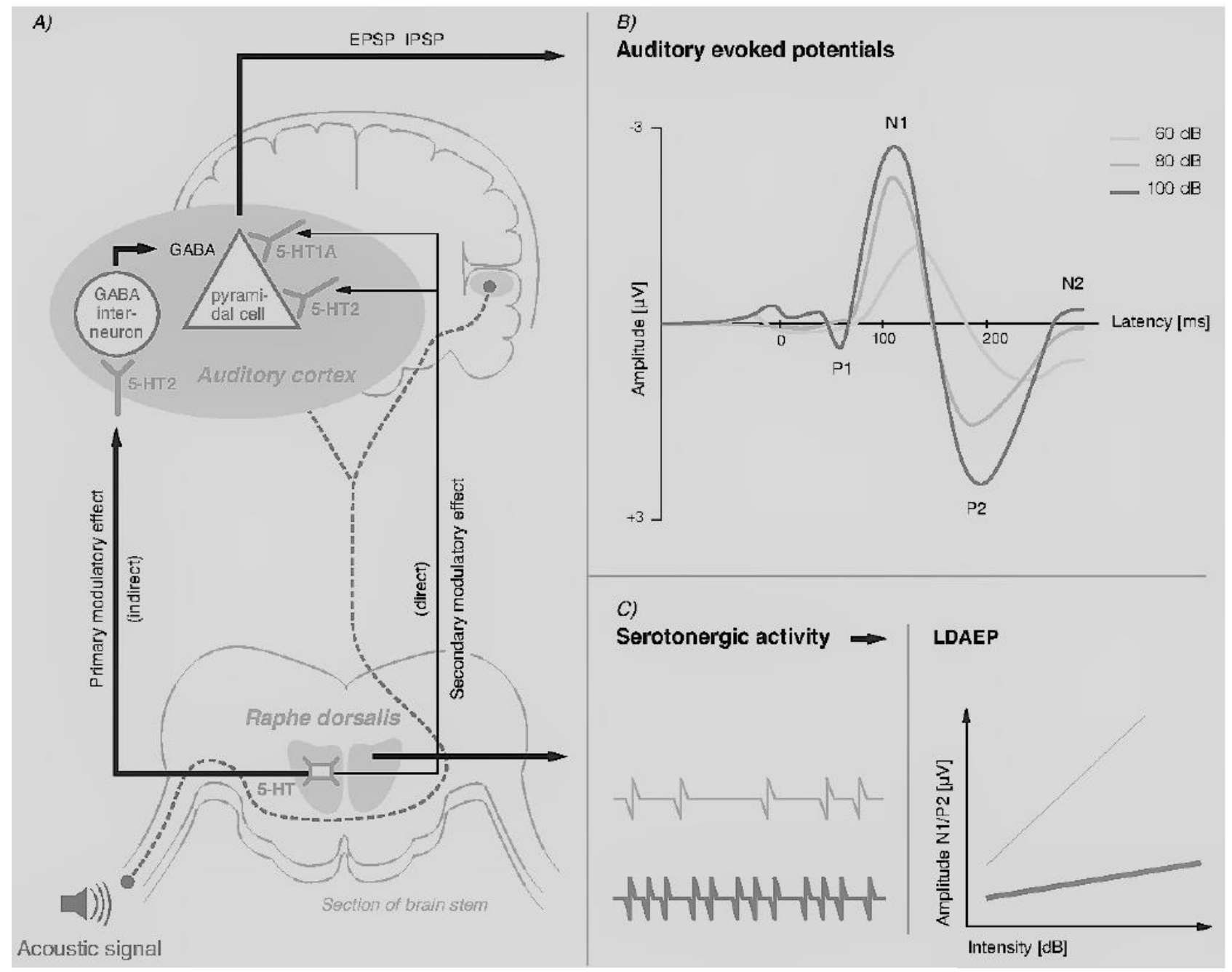

5-HT - serotonergic neurons, 5- HT1A - inhibitory presynaptic receptors, 5- HT2 - excitatory presynaptic receptors, EPSP/IPSP - excitatory- and inhibitory postsynaptic potentials. Adapted from Serotonin und akustisch evozierte Potentiale (p. 147), by G. Juckel, 2005, Darmstadt: Steinkopff Verlag. Copyright 2005 by Steinkopff Verlag Darmstadt.

Figure 2. The neurobiological model of the loudness dependence of the auditory evoked potential (LDAEP) in the primary auditory cortex 

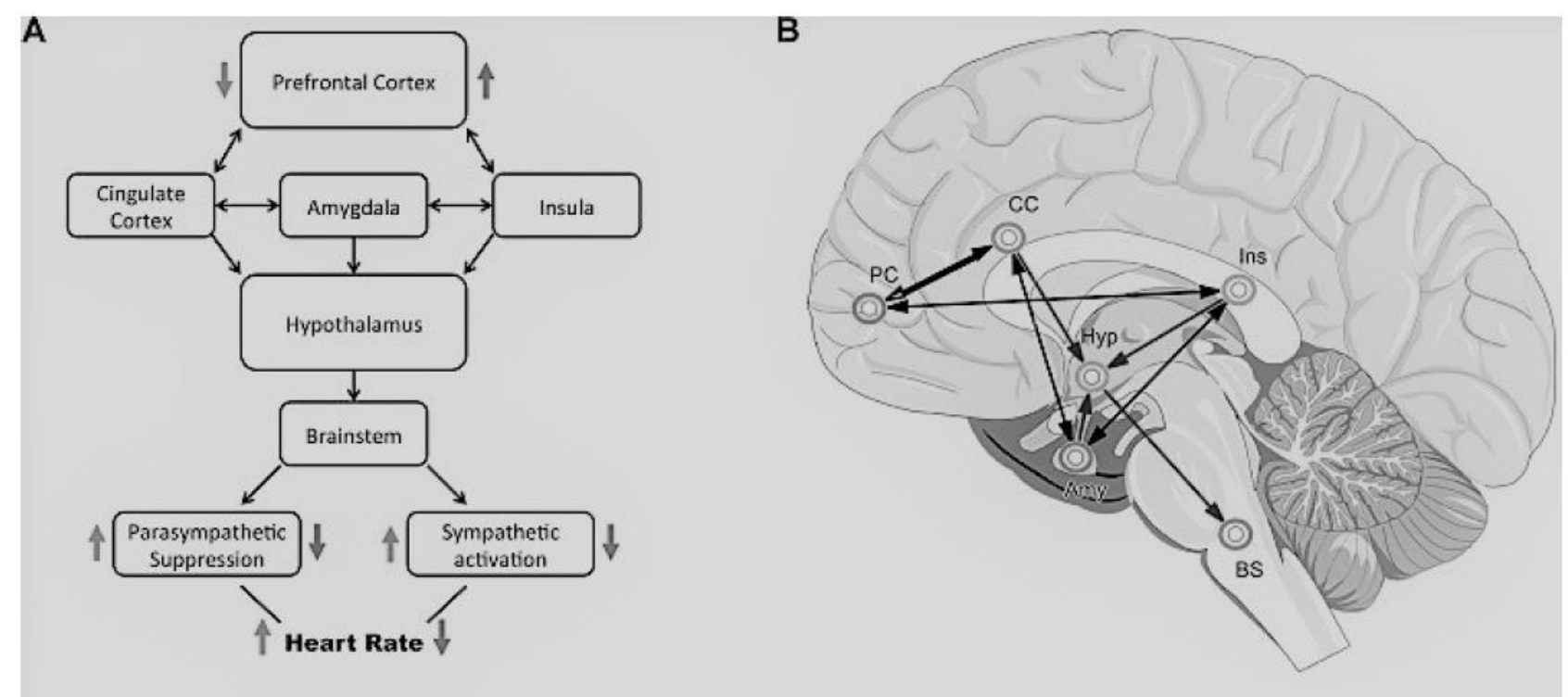

PC - prefrontal cortex, CC - cingulate cortex, Hyp - hypothalamus, Ins - insula, Amy - amygdala, BS - brainstem. Adapted from Nikolin et al. 2017. Copyright ( 2017 Nikolin et al

Figure 3. Corticolimbic regulation of hearth rhythm

\section{Heart rate variability}

Impaired flexibility of emotional regulation can also be investigated by heart rate variability - highfrequency heart rate variability and respiratory sinus arrhythmia - by measuring cardiac performance, which is also controlled by the cortico-limbic system (Myers 2016). Studies of high-frequency heart rate variability (Adolph et al. 2018, Wilson et al. 2016) and respiratory sinus arrhythmias (Tsypes et al. 2015, Yang et al. 2019) point to a reduced capacity to regulate the emotional response to stress in patients with suicidal symptoms. The central structures involved in the regulation of heart rhythm are shown in Figure 3.

\section{DISCUSSION}

Electrophysiological methods in contrast to functional and structure neuroimaging are easily replicable low-priced methods which can be part of any psychiatric institution. Furthermore with more advanced computer postprocessing methods and the application of a modern mathematical analysis they are even able to approach (functional) neuroimaging methods by a relative good spatial resolution and exceed them with their excellent time resolution. In approach to common clinical use in examination of suicidal patients there are different limits among these electrophysiological methods: the degree of cooperation of patient with potential tendency to dissimulation of suicidal symptoms limits especially event-related potentials, whereas polysomnographic examination requires specially trained personnel also during the night shift. Significant limitation of all electrophysiological research in the field of suicide is the often need (or even urgency) to medicate the patient at immediate suicidal risk, which also affects the quality and accuracy of the results in many cases, sometimes to an unacceptable extent (benzodiazepines!). The choice of a method for assessing suicidality also has significant implications for possibilities of comparison with other studies.

Reasons and examples which can explains conflicting conclusions of presented studies:

- various suicidal risk assessment - from a lifetime history of suicidal attempts - (Chen et al. 2005, Min et al. 2012, Tsypes et al. 2015) over examination by 1 item in Hamilton Depression Rating Scale (Iosifescu et al. 2010, Hunter et al. 2010, Roh et al. 2020, Bernert et al. 2017, Uhl et al. 2012, Graßnickel et al. 2015), over complex psychometric scales of suicidal risk such as The Columbia Suicide Severity Rating Scale (C-SSRS) (Wilson et al. 2016) to examination 3 weeks before committed suicide immediately after discharge from psychiatric hospital (Kopeček et al. 2007)

- EEG-affecting medication prior to testing - from no available data at all (Lee et al. 2017, Kim et al. 2011, Kudinova et al. 2016, Albanese et al. 2019, Tsypes et al. 2015, Yang et al. 2019) to missing exlusion of benzodiazepines (Pegg et al. 2018) and allowed using of benzodiazepines (Park et al. 2019)

- small files of suicidal subjects - from case study (Kopeček et al. 2007), to studies under 15 suicidal participants (Iosifescu et al. 2008, Hunter et al. 2010, Kim et al. 2019, Agargun et al. 2003, Kudinova et al. 2016, Tavakali et al. 2018), to only 2 studies over 50 suicidal participants (Lee et al. 2017, Albanus et al. 2019) and only 1 study over 100 suicidal participants (Arkina et al. 2019).

Taking into consideration all the above mentioned, nowadays it is probably not possible to point out the optimal electrophysiological examination that can be 
really useful in common medical practice for the "comprehensive" assessment of a suicidal patient. The most consistent and promising results are presented by evaluation of theta power by quantitative electroencephalography, although there are also few conflicting conclusions.

\section{CONCLUSION}

From a diagnostic and therapeutic point of view, a thorough clinical assessment of suicidal risk is important in suicidal patients. Equally (perhaps even more) important is the stratification of suicidality in research looking for a valid and readily available biomarker of suicidal symptoms. The authors of this paper believe that this article could be good starting point for further research of the pathomechanisms of the "suicidal brain" and in the search for the desired biomarker, it is appropriate to reach for more complex psychometric scales of suicidal risk such as The Columbia Suicide Severity Rating Scale and for the necessary anxiolysis or tranquillization of the patient choose medicaments with less potential to affect the electroencephalography.

\section{Acknowledgements: None.}

\section{Conflict of interest: None to declare.}

\section{Contribution of individual authors:}

Tomáš Rakús \& Katarína Hubčíková: participated mainly of design of the study, literature searches and analyses, statistical analyses, interpretation of data.

Lucia Bruncvik \& Zuzana Pechanová: participated mainly in writing manuscript.

Martin Brunovsky: was supervisor of all work.

\section{References}

1. Adolph $D$ et al.:High frequency heart rate variability: Evidence for a transdiagnostic association with suicide ideation. Biol Psychol. 2018;138:165-171

2. Agargun $M Y \&$ Cartwright R: REM sleep, dream variables and suicidality in depressed patients. Psychiatry Res. 2003;119: 33-39

3. Albanese BJ et al.: Impaired Conflict Detection Differentiates Suicide Attempters From Ideating Nonattempters: Evidence From Event-Related Potentials. Biol Psychiatry Cogn Neurosci Neuroimaging; 2019; 4: 902-912

4. Arikan MK et al.: A biological marker for suicide attempt in patients with depression. J Affect Disord; 2019; 254:1-6

5. Attal $Y \&$ Schwartz D: Assessment of subcortical source localisation using deep brain activity imaging model with minimum norm operators: An MEG study. In: PLoS ONE; 2013; 8

6. Baik SY et al.: ERP investigation of attentional disengagement from suicide-relevant information in patients with major depressive disorder. J Affect Disord 2018; 225:357-364
7. Ballard ED et al.: Nocturnal Wakefulness Is Associated With Next-Day Suicidal Ideation in Major Depressive Disorder and Bipolar Disorder. J Clin Psychiatry; 2016; 77: 825-831.

8. Bani-Fatemi A et al.: Structural and functional alterations of the suicidal brain: An updated review of neuroimaging studies. Psychiatry Res Neuroimaging; 2018; 278: 77-91.

9. Beck AT et al. : Assessment of Suicidal Intention: The Scale for Suicide Ideation. In: Journal of Consulting and Clinical Psychology; 1979; 47:343-352.

10. Bernert RA et al.: Sleep architecture parameters as a putative biomarker of suicidal ideation in treatmentresistant depression. J Affect Disord; 2017; 208: 309-315.

11. Boafo $A$ et al: Sleep architecture in adolescents hospitalized during a suicidal crisis. Sleep Medicine; 2019; 56: 41-46.

12. Bravermanová A et al: Auditivní evokované potenciály závislé na hlasitosti jako marker centrálni serotonergní transmise. Psychiatrie; 2016; 20:88-92.

13. Chen TJ et al.: Serotonin dysfunction and suicide attempts in major depressives: an auditory event-related potential study. Neuropsychobiology; 2005; 52: 28-36.

14. Cheng $P$ et al.: The role of fast and slow EEG aktivity during sleep in males and females with major depressive disorder. Psychophysiology; 2015; 52: 1375-1381.

15. Ciganek L:The EEG response (evoked potential) to light stimulus in man. Arch Ophthalmol; 1991; 109: 816-824.

16. Cook IA et al.: Assessing the accuracy of topographic EEG mapping for determining local brain function. In: Electroencephalogr. Clin Neurophysiol 1998; 107:408-414

17. Dolsen MR et al.: Neurophysiological correlates of suicidal ideation in major depressive disorder: Hyperarousal during sleep. J Affect Disord 2017; 212:160-166

18. Graßnickel $V$ et al.: Loudness dependence of auditory evoked potentials (LDAEP) in clinical monitoring of suicidal patients with major depression in comparison with non-suicidal depressed patients and healthy volunteers: A follow-up-study. J Affect Disord 2015; 184:299-304

19. Gross JJ \& John OP: Individual differences in two emotion regulation processes: implications for affect, relationships, and well-being. J Pers Soc Psychol.; 2003; 85: 348-62

20. Hegerl U \& Juckel G: Intensity dependence of auditory evoked potentials as an indicator of central serotonergic neurotransmission: a new hypothesis. Biol Psychiatry; 1993; 33:173-187.

21. Hegerl U et al.: Event-related potentials. Do they reflect central serotonergic neurotransmission and do they predict clinical response to serotonin agonists; $J$ Affect Disord; 2001;62:93-100.

22. Hensch T. et al.:Reliability of intensity dependence of auditory-evoked potentials. Clin Neurophysiol. Vol.; 2008; 119: 224-236.

23. Hughes JR \& John, ER: Conventional and quantitative electroencephalography in psychiatry. J Neuropsychiatry Clin Neurosci.; 1999; 11:190-208.

24. Hunter AM et al.: Brain functional changes (QEEG cordance) and worsening suicidal ideation and mood syptoms during antidepressant treatment. Acta Psychiatr Scand.; 2010; 122: 461-469.

25. Iosifescu DV et al.: Pretreatment frontal EEG and changes in suicidal ideation during SSRI treatment in major depressive disorder. Acta Psychiatr Scand; 2008; 117:271-276 
26. Jandl $M$ et al.:Suicide risk markers in major depressive disorder: a study of electrodermal activity and eventrelated potentials. J Affect Disord.; 2010; 123:138-149.

27. Jollant $F$ et al.:The suicidal mind and brain: a review of neuropsychological and neuroimaging studies. World $J$ Biol Psychiatry; 2010; 12: 319-339.

28. Juckel $G$ et al.:Auditory evoked potentials reflect serotonergic neuronal activity-a study in behaving cats administered drugs acting on 5-HT1A autoreceptors in the dorsal raphe nucleus. Neuropsychopharmacology.; 1998; 21: 710-716.

29. Juckel G: Serotonin und akustisch evozierte Potentiale, 147.Darmstadt: Steinkopff Verlag, 2005,

30. Kutcher S. \& Chehil S: Appendix 2 - Tool for Assessment of Suicide Risk (TASR) In: Kutcher S, Chehil S. Suicide Risk Management: A Manual for Health Professionals. 118. Oxford, Massachusetts, Carlton: Blackwell Publishing, 2007

31. Kopeček $M$ et al.:Can prefrontal theta cordance differentiate between depression recovery and dissimulation? Neuroendocrinol Lett 2007; 28: 524-526

32. Kim DH \& Park YM: The association between suicidality and serotonergic dysfunction in depressed patients. Journal of Affective Disorders 2013; 148:72-76.

33. Kim Y. et al.:What does electroencephalography coherence tell us about memory encoding in adolescents at high risk of suicide? Psychopathology 2019; 52:265-270

34. Kudinova $A Y$ et al.:Differences in emotion modulation using cognitive reappraisal in individuals with and without suicidal ideation: An ERP study. Cogn Emot.; 2016; 30: 999-1007

35. Lee SH et al.:Clinical implications of loudness dependence of auditory evoked potentials in patients with atypical depression. Prog Neuropsychopharmacol Biol Psychiatry; 2014; 54: 7-12.

36. Lee, SM et al.:Electroencephalographic correlates of suicidal ideation in the theta band. Clinical EEG and Neuroscience. ; 2017; 48: 316-321.

37. Lee HY et al.:Possible Association between Serotonin Transporter Gene Polymorphism and Suicide Behavior in Major Depressive Disorder. Psychiatry Investig.; 2015;12: 136-141.

38. Leonard WC et al.:Evaluation of veterans'suicide risk with use of linguistic detection methods. Psychiatr. Serv.; 2015; 66:1051-1056.

39. Leuchter AF et al.: Cordance: a new method for assessment of cerebral perfusion and metabolism using quantitative electroencephalography. Neuroimage.; 1994; 1: 208-219.

40. Mann JJ et al.: A serotonin transporter gene promoter polymorphism (5-HTTLPR) and prefrontal cortical binding in major depression and suicide. Arch Gen Psychiatry.; 2000; 57: 729-738.

41. Marsic A et al.:The Relationship Between Intentional Self? Injurious Behavior and the Loudness Dependence of Auditory Evoked Potential in Research Volunteers. J. Clin. Psychol.; 2015; 71:250-257.

42. Min JA et al.: Clinical characteristics associated with different strengths of loudness dependence of auditory evoked potentials (LDAEP) in major depressive disorder. Psychiatry Res.; 2012; 200: p. 374-381.

43. Myers B:Corticolimbic regulation of cardiovascular responses to stress. Physiology \& Behavior; 2016; 172:49-59
44. Nikolin $S$ et al.:Combined effect of prefrontal transcranial direct current stimulation and a working memory task on heart rate variability. PLOS ONE; 2017; 12.

45. Nofzinger EA et al.:Functional neuroimaging evidence for hyperarousal in insomnia.Am J Psychiatry; 2004; 161 : 2126-2128.

46. Pandey GN et al.:Higher expression of serotonin 5$H T(2 A)$ receptors in the postmortem brains of teenage suicide victims. Am J Psychiatry; 2002; 159: 419-429.

47. Park $Y$ et al.: Frontal alpha asymetry correlates with suicidal behavior in major depressive disorder. Clin Psychopharmacol Neurosci. ;2019; 17:377-387.

48. Park YM et al.:Serum BDNF Levels in Relation to Illness Severity, Suicide Attempts, and Central Serotonin Activity in Patients with Major Depressive Disorder: A Pilot Study. Plos One. ; 2014; 9.

49. Park YM:Relationship between Serotonergic Dysfunction Based on Loudness Dependence of Auditory-Evoked Potentials and Suicide in Patients with Major Depressive Disorder. Psychiatry Investig.; 2018; 12 : 421-424.

50. Pascual-Marqui RD et al.:Low resolution electromagnetic tomography: a new method for localizing electrical aktivity in the brain. J Psychophysiol.; 1994; 18: 49-65.

51. Pascual-Marqui RD, Esslen M, Kochi K et Lehmann D: Functional imaging with low-resolution brain electromagnetic tomography (LORETA): a review. Methods Find Exp Clin Pharmacol; 2002; 24 Suppl C:91-5

52. Pascual-Marqui RD : Standardized low-resolution brain electromagnetic tomography (sLORETA): technical details. Methods Find Exp Clin Pharmacol; 2002; 24 Suppl D:5-12.

53. Pascual-Marqui RD et al.: Assessing interactions in the brain with exact low-resolution electromagnetic tomography. Philos Trans A Math Phys Eng Sci; 2011: 3768-84.

54. Patterson LM et al.:5-HT radioligands for human brain imaging with PET and SPECT. Med Res Rev. ;2013; 33: 54-111.

55. Pegg SL et al.:Differentiating clinically depressed adolescents with and without active suicidality: An examination of neurophysiological and self-report measures of reward responsiveness. Depression and Anxiety. 2020; 1-9.

56. Perlman CM et al.: Inventory of suicide risk assessment tools. In: Perlman CM, Neufeld E, Martin L, Goy M, Hirdes JP. Suicide risk assessment guide: a resource for health care organizations. 35-64. Toronto: Ontario Hospital Association and Canadian patient safety institute 2011

57. Posner K et al.: The Columbia - Suicide Severity Rating Scale: Initial validity and internal consistency findings from three multisite studies with adolescents and adults. The American Journal od Psychiatr; 2011; 168:12661277

58. Rajappa $K$ et al.:Eotion Dysregulation and Vulnerability to Suicidal Ideation and Attempts. Cogn Ther Res.; 2012; 36: 833-839.

59. Randall JR et al.: Predicting Future Suicide: Clinician Opinion versus a Standardized Assessment Tool. Suicide Life Threat Behav ; 2018; 49: 941-951.

60. Roh SCH et al.:Frontal alpha asymmetry moderated by suicidal ideation in patients with major depressive disorder: A comparison with healthy individuals. Clin Psychopharmacol Neurosci.; 2020; 18: 58-66. 
61. Schiller MJ: Quantitative Electroencephalography in Guiding Treatment of Major Depression. Frontiers in Psychiatry 2019; 9:1-7

62. Song $W$ et al.: Effect of Affective Reward on Cognitive Event-related Potentials and its Relationship with Psychological Pain and Suicide Risk among Patients with Major Depressive Disorder. Suicide Life Threat Behav 2019; 49:1290-1306

63. Tavakoli $P$ et al.: Event-Related Potential Measures of Attention Capture in Adolescent Inpatients With Acute Suicidal Behavior. Front Psychiatry 2018; 85

64. Thatcher $R W$ et al.:Cortico-cortical associations and EEG coherence: A two-compartmental model. Electroencephalography and Clinical Neurophysiology 1986; 64:23-143

65. Tsypes $A$ et al.: Resting respiratory sinus arrhythmia in suicide attempters. Psychophysiology 2018; 55
66. Uhl I et al.: Loudness dependence of auditory evoked potentials (LDAEP) in clinical monitoring of suicidal patients with major depression: a pilot study. Eur Arch Psychiatry Clin Neurosci 2012;262:487-492

67. Wenzel A \& Beck AT: A cognitive model of suicidal behavior: Theory and treatment. Applied and Preventive Psychology 2008; 12:189-201

68. Wilson ST et al.: Heart rate variability and suicidal behavior. Psychiatry Res 2016; 240:241-247

69. WHO. World Health Organisation: Suicide Prevention (SUPRE). 2007. http://www.who.int/entity/mental_health/ management/en/SUPRE flyerlpdf?.ua $=1$

70. YANG X. et al.: Autonomic correlates of lifetime suicidal thoughts and behaviors among adolescents with a history of depression. Psychophysiology; 2019; 56

Correspondence:

Tomáš Rakús, $M D$

Department of neuropsychiatry of Slovac Medical University in Bratislava and Psychiatric Hospital of Philippe Pinel in Pezinok, Slovakia; Third faculty of medicine Charles University in Prague, Czech republic

Malacká cesta 63, 90218 Pezinok, Slovakia

E-mail: tomas.rakus.1973@gmail.com 